\title{
Microwave Imaging via Space-Time Beamforming: Experimental Investigation of Tumor Detection in Multilayer Breast Phantoms
}

\author{
Xu Li, Member, IEEE, Shakti K. Davis, Student Member, IEEE, Susan C. Hagness, Member, IEEE, \\ Daniel W. van der Weide, Member, IEEE, and Barry D. Van Veen, Fellow, IEEE
}

\begin{abstract}
Microwave imaging via space-time (MIST) beamforming has been proposed recently for detecting small malignant breast tumors. In this paper, we extend the previously presented two-dimensional space-time beamformer design to three-dimensional (3-D), and demonstrate its efficacy using experimental data obtained with a multilayer breast phantom. The breast phantom consists of a homogeneous normal breast tissue simulant covered by a thin layer of skin simulant. A small synthetic malignant tumor is embedded in the breast phantom. We have developed several tumor simulants that yield the range of dielectric contrasts between normal and malignant tissue that are expected in clinical scenarios. A microwave sensor comprised of a synthetic planar array of compact ultrawide-band (UWB) antennas is immersed in a coupling medium above the breast tissue phantom. At each position in the array, the antenna transmits a synthetically generated pulse (1-11 GHz) into the phantom. The received backscatter signals are processed by a data-adaptive algorithm that removes the artifact caused by antenna reverberation and backscatter from the skin-breast interface, followed by 3-D space-time beamforming to image backscattered energy as a function of location. Our investigation includes a numerical (finite difference time domain) and experimental study of the UWB antenna performance in the immersion medium, as well as a study of the influence of malignant-to-normal breast tissue dielectric contrast on dynamic range requirements and tumor detectability. This paper represents the first experimental demonstration of 3-D MIST beamforming in multilayer breast phantoms with malignant-to-normal dielectric contrasts down to $1.5: 1$ for a 4-mm synthetic tumor.
\end{abstract}

Index Terms-Breast cancer detection, finite difference time domain (FDTD), microwave imaging, space-time beamforming, tissue phantoms, ultrawide-band (UWB) antennas.

\section{INTRODUCTION}

$\mathbf{E}$ ARLY detection and timely medical intervention are key factors affecting long-term survival and life quality of breast-cancer patients. X-ray mammography remains the pri-

Manuscript received September 19, 2003; revised March 13, 2004. This work was supported by the Department of Defense Breast Cancer Research Program under Award DAMD17-02-1-0625, by the National Science Foundation under Grant BES 0201880, by the National Institutes of Health under Grant R21 CA92188-01 awarded by the National Cancer Institute, and under the Graduate Engineering Research Scholars Program.

$\mathrm{X}$. Li is with the Department of Biomedical Engineering, Northwestern University, Evanston, IL 60208 USA (e-mail: xuli@ northwestern.edu).

S. K. Davis, S. C. Hagness, D. W. van der Weide, and B. D. Van Veen are with the Department of Electrical and Computer Engineering, University of Wisconsin-Madison, Madison, WI 53706 USA (e-mail: skdavis@ cae.wisc.edu; hagness@engr.wisc.edu; danvdw@engr.wisc.edu; vanveen@engr.wisc.edu).

Digital Object Identifier 10.1109/TMTT.2004.832686 mary screening method for detecting nonpalpable early-stage breast tumors. However, despite significant progress in improving the mammographic technique, well-recognized limitations persist [1]. Most significantly, there is a need for improved sensitivity and specificity, particularly in the case of radiographically dense breast tissue. Approximately 4\%-34\% of all breast cancers are missed by conventional mammography [2], while nearly $70 \%$ of all breast lesions identified by mammography turn out to be benign [3]. Other drawbacks of mammography include the risk of accumulating low-dose ionizing radiation over repeated scans and patient discomfort due to breast compression during the exam. These limitations motivate the search for alternative or complementary technologies for early breast cancer detection. One such modality is microwave imaging.

Both passive and active microwave-imaging techniques are under investigation for breast cancer detection [4]. Passive microwave radiometry [5], [6] exploits temperature differences between malignant and normal breast tissue. Active microwave imaging exploits the significant dielectric contrast between malignant tumors and normal breast tissue at microwave frequencies ([7]-[9]), which results in microwave scattering or selective heating/absorption. The dielectric contrast, which is estimated to be greater than $2: 1$, is much higher than the few percent contrast in radiographic density exploited by X-ray mammography. Therefore, even though active microwave imaging does not offer the high spatial resolution provided by X-rays, it has the potential to offer improved sensitivity and specificity. In addition, active microwave imaging is a nonionizing and noninvasive modality, and does not require breast compression. The radiated power level is much lower than that from a typical cell-phone antenna.

Three types of active microwave breast imaging techniques have been proposed: hybrid microwave-induced acoustic imaging [10], [11], microwave tomography [12]-[16], and ultrawide-band (UWB) microwave radar techniques [17]-[23]. In the hybrid approach, microwave signals are transmitted into the breast to heat tumors, and ultrasound transducers detect pressure waves generated by tumor expansion. The received waveforms are used to infer the tissue dielectric-properties distribution inside of the breast. In tomographic image reconstructions, a nonlinear inverse scattering problem is solved to recover the spatial distribution of dielectric properties in the breast. In contrast to tomography, the proposed UWB radar 
approaches seek only to identify the presence and location of significant backscattered energy from malignant breast tumors.

Our recently proposed method of microwave imaging via space-time (MIST) beamforming [22], [23] is representative of the class of UWB radar techniques. The space-time beamformer assumes that each antenna in an array sequentially transmits a low-power UWB signal into the breast and records the backscatter. The backscatter signals are passed through the beamformer, which is designed to image backscattered energy as a function of location in the breast. Malignant tumors produce localized regions of relatively large backscatter energy due to their significant dielectric contrast with normal breast tissue and, thus, stand out in the image. Note that the current scope of this approach is the detection of abnormalities inside of the breast. Since certain types of benign breast lesions may also exhibit a significant dielectric contrast relative to normal breast tissue, differentiation between malignant and benign tumors will likely involve the examination of other scattering characteristics such as polarization and spectral signatures [18], and is beyond the scope of this paper.

We previously demonstrated the theoretical feasibility of MIST beamforming for tumor detection by applying two-dimensional (2-D) space-time beamformers to simulated backscatter data obtained from a variety of anatomically realistic MRI-derived 2-D finite-difference time-domain (FDTD) breast models [22], [23]. The results show that the MIST beamforming approach offers significant improvements over previous UWB radar techniques based on simpler focusing schemes [17], [19]-[21]. The robustness of MIST beamforming with respect to a number of potential challenges associated with imaging the inherently heterogeneous breast has also been demonstrated.

While extensive experimental results have been obtained using a pre-clinical prototype of a microwave tomographic system [13], only very preliminary experimental studies have been reported to date using UWB radar techniques. In a recent experimental feasibility study [24], simple time-shift-and-sum focusing schemes [17], [19]-[21] were used to detect a 2-D wood, copper, or water-filled object (representing a malignant tumor) inside an otherwise hollow PVC pipe (representing skin and normal breast) in free space. In this study, the pipe was illuminated by a large horn antenna or resistively loaded monopole antenna positioned at several points encircling the pipe. The study was designed to mimic the system configuration where the patient is lying in a prone position with antennas surrounding the breast. In our own initial experimental feasibility study of the MIST beamforming approach [25], small water-based synthetic malignant tumors were successfully imaged in a homogeneous 3-D background. This first-generation phantom did not contain a layer of skin and the dielectric contrast between the normal and malignant-tissue simulants was somewhat larger than that measured in actual breast tissue.

In this paper, we present an in-depth experimental study of the MIST beamforming approach using second-generation multilayer 3-D breast phantoms. In contrast to our previous research, our new class of breast phantoms now features a thin layer of skin simulant that separates the interior of the breast phantom from the antenna immersion medium, and normal and malignant tissue simulants that mimic the dielectric contrast expected in clinical scenarios. The experimental configuration is designed to mimic a scan of a patient lying in a supine position. A microwave sensor comprised of a mechanically scanned UWB antenna is immersed in a coupling medium above the breast phantom. At each position in the array, the antenna transmits a synthetically generated pulse into the phantom. The received backscatter signals are processed by a data-adaptive algorithm that removes the artifact caused by antenna reverberation and backscatter from the skin-breast interface. We extend the previously reported 2-D beamformer design and implementation [22], [23] to three-dimensional (3-D), and demonstrate its efficacy using the experimental data. Our investigation includes a study of the performance of the UWB antenna in the immersion medium. We obtain excellent agreement between the experimental results and FDTD simulations [26] of the antenna. The imaging results demonstrate that the MIST beamforming approach offers the potential of detecting small $(<5 \mathrm{~mm})$ breast tumors with realistic dielectric contrasts with respect to normal breast tissue using state-of-the-art, but readily available hardware.

Section II reviews published measurements of breast-tissue dielectric properties. These measurement results provide the basis for the range of dielectric contrasts selected for our experimental breast phantoms. The phantom configuration and experimental setup are described in Section III. Section IV provides a detailed numerical and experimental characterization of the UWB antenna element that is used to transmit and receive microwave signals. Section V presents the signal-processing algorithms with emphasis on the introduction of the 3-D MIST beamforming procedure, as well as representative imaging results. In Section VI, we investigate the impact of dielectric contrast on system dynamic range requirements and the image signal-to-clutter ratios.

\section{REVIEW OF BREASt Tissue DiElectric PROPERTIES}

Several dielectric spectroscopy studies reported in the literature over the past 20 years suggest that the dielectric-properties contrast between malignant and normal breast tissue is greater than $2: 1$ in the RF and microwave frequency range. Recognition of the potential diagnostic value of such a contrast is one of the primary motivating factors for the development of active microwave imaging technologies for breast cancer detection. The fundamental difference in the permittivity and conductivity of breast carcinoma and normal breast tissues appears to arise in part from the increased water content in neoplastic tissue due to increased protein hydration [27] and vascularization/angiogenesis of malignant tumors. Thus, microwave frequencies can exploit potentially strong indicators of malignancy associated with physical or physiological factors of clinical interest.

Breast-tissue dielectric properties published by Chaudhary et al. [7], Surowiec et al. [8], and Joines et al. [9] are summarized in Fig. 1 as a function of frequency. For breast imaging, we are interested in the $1-10-\mathrm{GHz}$ regime because it appears to balance the conflicting demands of better spatial resolution (higher frequencies) and better penetration depth (lower frequencies). We note that the existing 


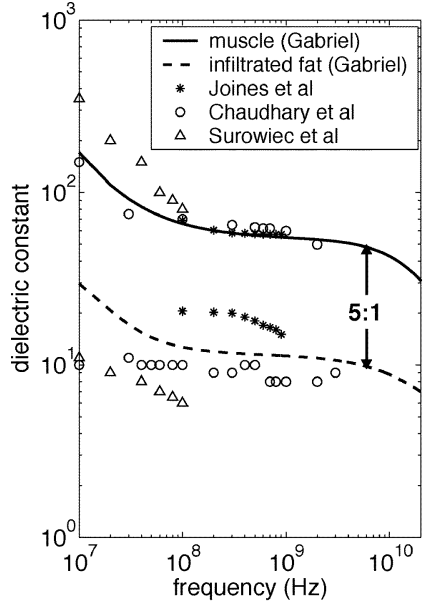

(a)

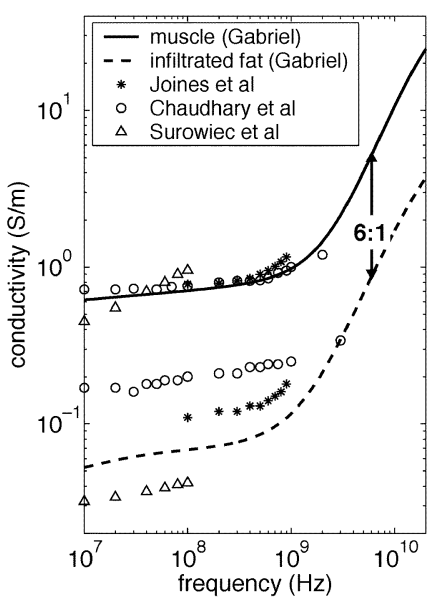

(b)
Fig. 1. Summary of measured dielectric-properties data for normal and malignant breast tissue at radio and microwave frequencies. Four-term Cole-Cole parametric dispersion models for infiltrated fat and muscle are used to illustrate the extrapolation of measured data to higher frequencies (above $3 \mathrm{GHz})$.

studies on normal and malignant breast tissue do not cover frequencies above $3 \mathrm{GHz}$. Therefore, in Fig. 1, we extrapolate the measured data to higher frequencies using established four-term $(10 \mathrm{~Hz}-100 \mathrm{GHz})$ Cole-Cole parametric dispersion models [28] for the complex permittivity of infiltrated fat and muscle-two biological tissues that mimic the lower and upper bounds on the relative permittivity $\left(\epsilon_{r}\right)$ and conductivity [ $\sigma$ (in siemens per meter)] of tissue in the breast

$$
\epsilon_{r}-j \frac{\sigma}{\omega \epsilon_{0}}=\epsilon_{\infty}+\sum_{n=1}^{4} \frac{\Delta \epsilon_{n}}{1+\left(j \omega \tau_{n}\right)^{\left(1-\alpha_{n}\right)}}-j \frac{\sigma_{s}}{\omega \epsilon_{0}} .
$$

The parameters for infiltrated fat $\left(\epsilon_{\infty}=2.5, \sigma_{s}=0.035 \mathrm{~S} / \mathrm{m}\right.$, $\Delta \epsilon_{1}=9.0, \Delta \epsilon_{2}=35, \Delta \epsilon_{3}=3.3 \times 10^{4}, \Delta \epsilon_{4}=1.0 \times 10^{7}$, $\tau_{1}=7.96 \mathrm{ps}, \tau_{2}=15.92 \mathrm{~ns}, \tau_{3}=159.15 \mu \mathrm{s}, \tau_{4}=15.915 \mathrm{~ms}$, $\alpha_{1}=0.20, \alpha_{2}=0.10, \alpha_{3}=0.05, \alpha_{4}=0.01$ ) yield $\epsilon_{r}=9.8$ and $\sigma=0.87 \mathrm{~S} / \mathrm{m}$ at $6 \mathrm{GHz}$, while the parameters for muscle $\left(\epsilon_{\infty}=4, \sigma_{s}=0.2 \mathrm{~S} / \mathrm{m}, \Delta \epsilon_{1}=50, \Delta \epsilon_{2}=7.0 \times 10^{3}, \Delta \epsilon_{3}=\right.$ $1.2 \times 10^{6}, \Delta \epsilon_{4}=2.5 \times 10^{7}, \tau_{1}=7.23 \mathrm{ps}, \tau_{2}=353.68 \mathrm{~ns}, \tau_{3}=$ $318.31 \mu \mathrm{s}, \tau_{4}=2.274 \mathrm{~ms}, \alpha_{1}=0.10, \alpha_{2}=0.10, \alpha_{3}=0.10$, $\alpha_{4}=0.0$ ) yield a dielectric constant of 48.2 and conductivity of $5.2 \mathrm{~S} / \mathrm{m}$ at $6 \mathrm{GHz}$. This data extrapolation process suggests a baseline contrast between malignant and normal breast tissue on the order of $5: 1$ in dielectric constant and $6: 1$ in conductivity. We are currently conducting dielectric spectroscopy measurements on freshly excised breast tissue specimens at microwave frequencies (up to $20 \mathrm{GHz}$ ) in a collaborative research effort between the University of Wisconsin-Madison and the University of Calgary, Calgary, AB, Canada. The higher-frequency extrapolations of the data in Fig. 1 are supported by our preliminary dielectric characterization data.

The variability in the dielectric properties of normal breast tissue reported in any individual study is less than $\pm 10 \%$ [7], [9]. However, the different dielectric properties data sets are not all in agreement, as illustrated by the variability in normal breast tissue properties across the three studies summarized in Fig. 1. Furthermore, our own measurements to date indicate that the

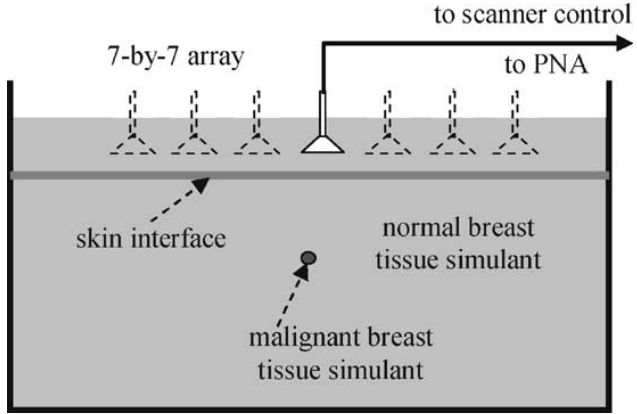

Fig. 2. Schematic showing a cross-sectional side view of the experimental setup.

impact of the heterogeneity of normal breast tissue on dielectric properties is more significant than the variability suggested by any of the individual studies of Fig. 1. The heterogeneity of normal tissue may, in fact, explain the variability observed across the different studies in Fig. 1. In addition, the average dielectric properties of normal breast tissue may vary considerably from patient to patient due to differences in the amount of fat versus fibroglandular tissue. Increased average density or heterogeneity of breast tissue will result in a reduced contrast with malignant tissue. For example, Meaney et al. [13] recently reconstructed the dielectric properties of normal breast tissue in vivo using a clinical prototype of a microwave tomographic system operating at $900 \mathrm{MHz}$. Their reconstructed average permittivity and conductivity profiles of normal breast tissue are considerably higher than the previously published values measured ex vivo by open-ended coaxial probes, suggesting that the contrast between normal and malignant breast tissue may be closer to $2: 1$.

Due to the uncertainty of the dielectric contrast between malignant and normal breast tissue, we consider contrasts in $\varepsilon_{r}$ ranging from approximately $5: 1$ down to less than $2: 1$ in this paper. The scenarios where the dielectric contrast is reduced from the $5: 1$ baseline case are inherently more challenging for tumor detection and, therefore, are good tests of the robustness of our imaging method.

\section{Multilayer BReast Phantom CONFiguration AND EXPERIMENTAL SETUP}

The experiment setup shown in Fig. 2 emulates a system configuration where a patient is lying in a supine position with a 2-D antenna array placed near the surface of the naturally flattened breast. The breast phantom consists of a container filled with a liquid mimicking normal breast tissue, a small synthetic tumor suspended in the liquid, and a thin layer of material representing the skin layer covering the normal breast tissue simulant. A single UWB antenna is sequentially repositioned in the horizontal plane using a computer-controlled mechanical $x-y$ scanner to synthesize a 2-D antenna array placed above the skin. The antenna is immersed in a matching medium to couple microwave energy into the breast more efficiently. Here, for simplicity, the liquid used for normal breast tissue simulant is also used as the immersion medium.

The tissue simulants in the phantom are chosen to approximate the dielectric properties of the corresponding tissue types. 
Most importantly, the dielectric contrast between the tissue simulants mimics the contrasts observed between different biological tissues at microwave frequencies. In addition to dielectric properties, factors such as availability, cost, toxicity, and stability are taken into consideration in choosing phantom materials. In the study presented in this paper, soybean oil is used as the normal breast tissue simulant because it is an inexpensive nontoxic liquid with dielectric properties roughly similar to very low-water-content fatty tissue. The soybean oil is contained in a $36 \mathrm{~cm} \times 36 \mathrm{~cm} \times 26 \mathrm{~cm}$ tank. The tank is large enough so any reflection from the boundaries or bottom of the tank can be removed using time gating. The dielectric properties of the oil $\left(\varepsilon_{r}=2.6\right.$ and $\sigma=0.05 \mathrm{~S} / \mathrm{m}$ at $6 \mathrm{GHz}$ ), as measured using an open-ended coaxial probe technique [29], fall slightly below the expected range of the dielectric properties for fatty breast tissue. Therefore, we have chosen materials for the skin and tumor simulants that similarly underestimate the actual dielectric properties of those tissue types so that the dielectric contrasts in the breast phantom are representative of those for actual tissue.

Since a large volume of normal breast tissue simulant is required in this experimental setup, the variation of malignant-to-normal tissue contrast is introduced by changing the dielectric properties of the malignant tissue simulant while using the same normal tissue simulant. Five malignant tissue simulants with varying $\varepsilon_{r}$ and conductivity $\sigma$ are developed using a diacetin-water solution with different concentrations. The resulting contrast in $\varepsilon_{r}$ between malignant and normal tissue simulants ranges from $1.5: 1$ to $5.2: 1$. As discussed in Section II, this represents the range of contrasts expected in clinical scenarios and is representative of likely between-patient variability in average breast density. Fig. 3(a) plots $\varepsilon_{r, \text { malignant }} / \varepsilon_{r, \text { normal }}$ as a function of water content in the malignant tissue simulants. As the diacetin solution is diluted with more water, the mixture exhibits an increased $\varepsilon_{r}$ and $\sigma$ and, thus, yields a higher dielectric contrast with the normal breast tissue simulant. Fig. 3(b) shows the measured dielectric constant of normal and malignant tissue simulants for the entire frequency range of interest $(1-11 \mathrm{GHz})$. The synthetic tumor is made by pouring the water-diacetin mixture into a 4-mm-diameter cylindrical container that has a height of $4 \mathrm{~mm}$. The container is composed of a section of plastic tube wrapped and sealed with a latex membrane. The dielectric properties of the container materials are similar to those of the soybean oil. A 0.1-mm-diameter nylon thread is used to suspend the synthetic tumor in the oil.

The skin layer in the phantom is created using a 1.5 -mm-thick unclad FR4 glass epoxy printed circuit board (PCB). According to the manufacturer, the dielectric properties of FR4 at $1 \mathrm{GHz}$ are $\varepsilon_{r}=4.34$ with a loss tangent of 0.016 . Thus, as desired, the dielectric constant of the skin simulant falls in between that of the normal and malignant tissue simulants.

During data collection, the UWB antenna is sequentially scanned in 1-cm increments to 49 different positions in a $6 \mathrm{~cm}$ $\times 6 \mathrm{~cm}$ array. The antenna element is positioned so that its aperture is $1 \mathrm{~cm}$ above the skin surface. The antenna is connected to an Agilent E8364A (10 MHz-50 GHz) performance network analyzer (PNA) to transmit and receive microwave signals. At each antenna location in the synthetic array, the

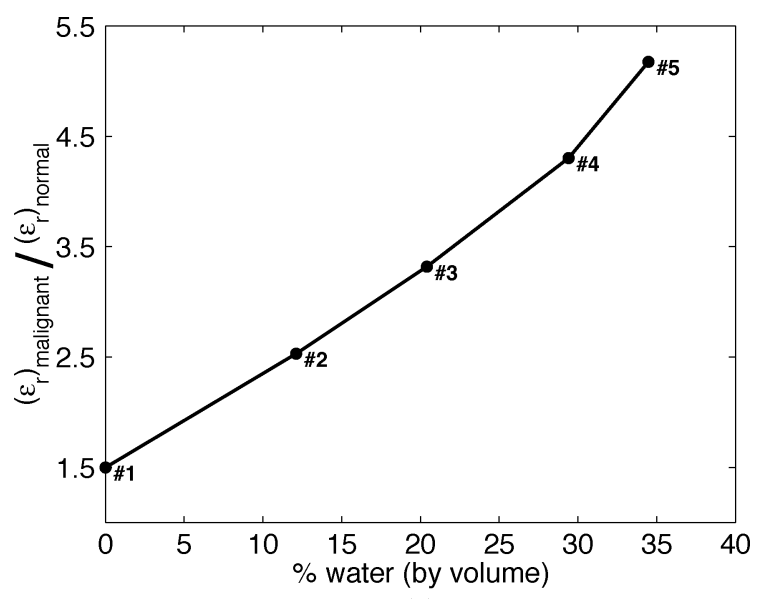

(a)

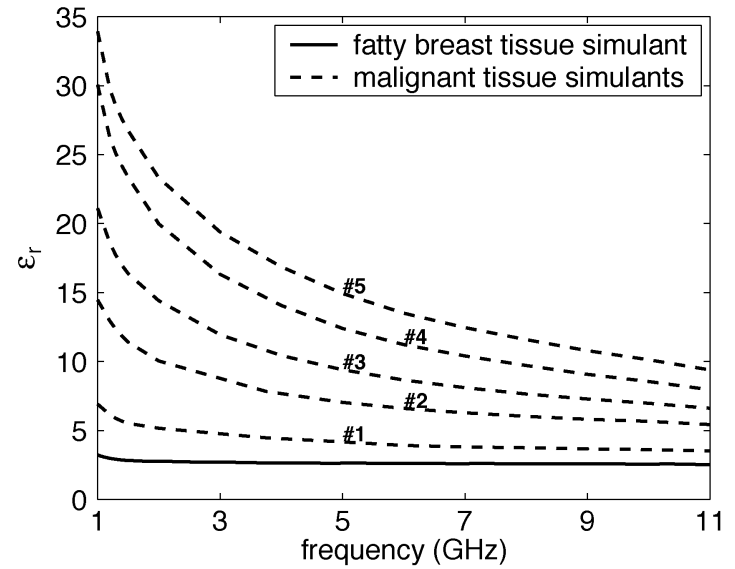

(b)

Fig. 3. (a) Contrast in $\varepsilon_{r}$ at $6 \mathrm{GHz}$ between normal and malignant breast tissue simulants. The horizontal axis shows the percentage of water (by volume) present in the water-diacetin solution used for the malignant tissue simulants. (b) Measured $\varepsilon_{r}$ of the normal breast tissue simulant and the five different malignant breast tissue simulants as a function of frequency.

PNA performs a frequency sweep from 1 to $11 \mathrm{GHz}$ with 201 frequency samples and records the backscatter $\left(S_{11}\right.$-parameter). The frequency-domain backscattered signals are scaled by the spectrum of the desired input pulse and transformed to the time domain using an inverse fast Fourier transform (FFT) algorithm. In the results presented in this paper, the input is a modulated Gaussian pulse given by

$$
e(t)=e^{-\frac{(t-4 \tau)^{2}}{\tau^{2}}} \sin \left(2 \pi f_{0}(t-4 \tau)\right)
$$

where $f_{0}=6 \mathrm{GHz}$ and $\tau=80 \mathrm{ps}$. The spectrum of this source waveform has a peak near $6 \mathrm{GHz}$ and a $1 / e$ bandwidth of $8 \mathrm{GHz}$, which is sufficiently covered by the $1-11-\mathrm{GHz}$ swept frequency range.

\section{DESIGN AND Characterization OF THE UWB ANTENNA}

The UWB antenna used for transmitting/receiving microwave energy is a modified version of a double-ridged pyramidal horn antenna [30], [31]. The waveguide section is eliminated, and one of the two ridges is replaced by a curved metallic plane terminated by resistors. The generic form of this configuration has been proposed in [32]. We customized this design to centimeter-scale dimensions for operation in the 


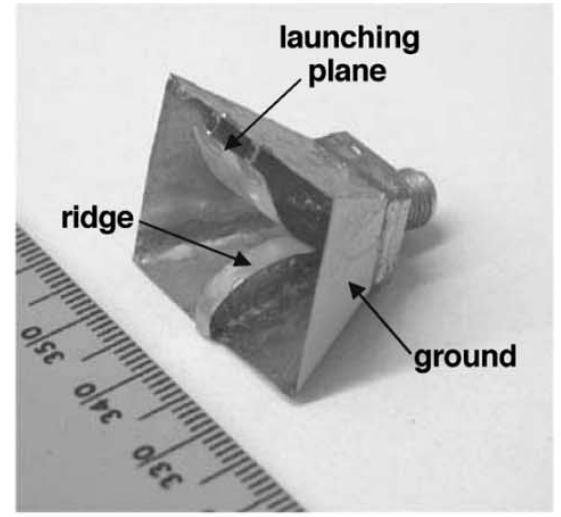

(a)

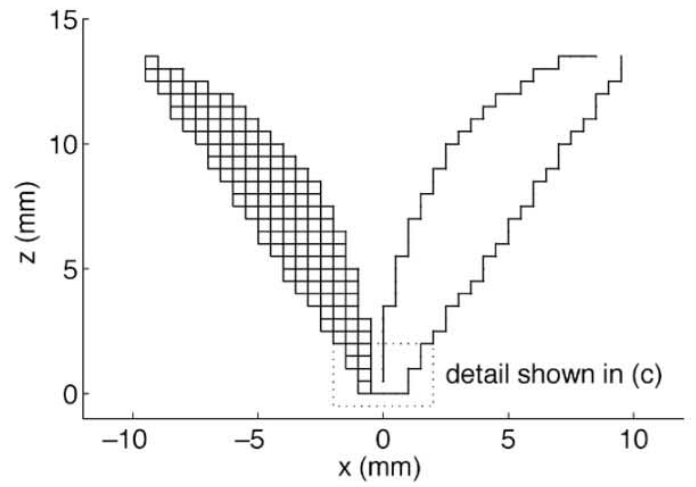

(b)

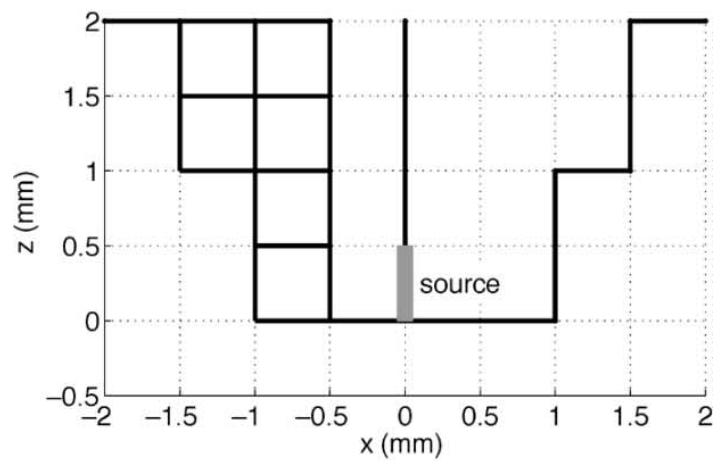

(c)

Fig. 4. (a) UWB antenna used in the microwave imaging system. (b) FDTD model of the UWB antenna geometry (cross-sectional side view with the launching plane on the right-hand side). The solid lines depict electric field vector components located along metal surfaces. The region bounded by the dashed rectangle is zoomed in in (c) to illustrate the geometry of the antenna feed. (c) Cross-sectional side view of the antenna feed model.

microwave frequency range and characterized its time- and frequency-domain performance in free space [33]. Here, we present an experimental and numerical time-domain characterization of the UWB antenna immersed in the phantom's coupling medium (soybean oil).

As shown in Fig. 4(a), the main structure of the antenna includes a pyramidal horn radiation cavity, metallic ridge, and curved metallic launching plane terminated with resistors. The pyramidal horn is connected to the outer conductor of the coaxial feed via a subminiature A (SMA) connector and serves as the ground plane providing a current return path. The depth of the pyramidal horn is $1.3 \mathrm{~cm}$ and the aperture dimensions are $2.5 \mathrm{~cm} \times 2.0 \mathrm{~cm}$. The curved planar launching plane is connected to the central conductor of the coaxial feed. It curves toward one of the sidewalls of the pyramidal horn and tapers toward the feed point. Two $100-\Omega$ termination resistors are connected in parallel near opposite corners of the launching plane to suppress reflections from the end of the launching plane. A ridge is attached to the interior side of the horn-shaped ground plane opposite the curved launching plane. The top surface of the ridge curves from the feed point toward the antenna aperture.

We developed a 3-D FDTD model of the UWB antenna to assist in the characterization of the antenna. The antenna geometry is modeled using a uniform space lattice with $0.5-\mathrm{mm}$ grid resolution. The curved or flared metal surfaces of the antenna are modeled using a staircased approximation. Fig. 4(b) displays a 2-D cut through the FDTD antenna model with the launching plane positioned on the right-hand side. The solid lines depict electric field vector components located along metal surfaces. Fig. 4(c) shows an enlarged view of the feed geometry. The base of the pyramidal horn is covered by a small metal plane to provide a complete ground plane. A vertical gap of one grid cell exists between the feed point of the launching plane and the base of the horn. The antenna excitation is implemented using a $1.0-\mathrm{V}$ $50-\Omega$ resistive voltage source across the gap [34]. The $100-\Omega$ termination resistors attached to the end of the launching plane are incorporated into the FDTD model using a lumped circuit element formulation [34]. The FDTD grid is terminated with a Berenger perfectly matched layer absorbing boundary condition [35].

The UWB performance of the antenna can be characterized in the time domain by the fidelity $(F)$, which is a measure of how accurately the transmitted waveform reproduces the time derivative of the voltage applied to the antenna terminals or, equivalently, how accurately the received voltage reproduces the transient field incident upon the antenna [36]. To investigate the antenna's fidelity in transmission and reception of the UWB signal in the immersion medium, two replicas of the antenna shown in Fig. 4 are connected to the two ports of the PNA and aligned face-to-face immersed in soybean oil with a 5-cm separation between the ends of the pyramidal horns. In the FDTD simulation, the two antennas are modeled using a configuration similar to the experimental setup. Using the procedures described in [33], we apply the source waveform plotted in Fig. 5(a) to the input terminals of the transmitting antenna. Fig. 5(b) shows the simulated and measured waveforms recorded at the receiving antenna. The fidelity, defined as $F=\max _{\tau} \int_{-\infty}^{\infty} \hat{r}(t-\tau) \hat{f}(t) d t$, corresponds to the maximum magnitude of the cross-correlation between the normalized observed response $\hat{r}(t)$ and ideal response $\hat{f}(t)$. A fidelity of $F=1$ indicates a perfect match between $\hat{r}(t)$ and $\hat{f}(t)$. Here, $\hat{f}(t)$ is calculated as the normalized time derivative of the source waveform, and $\hat{r}(t)$ is calculated using the normalized versions of the simulated and measured waveforms plotted in Fig. 5(b). This calculation yields a fidelity of approximately 0.94 and 0.97 for measured and simulated data, respectively, which verifies the excellent agreement between the transmitted and received waveforms shown in Fig. 5(b).

To examine the spatio-temporal radiation characteristics of the antenna, we use FDTD simulations to compute the transient 


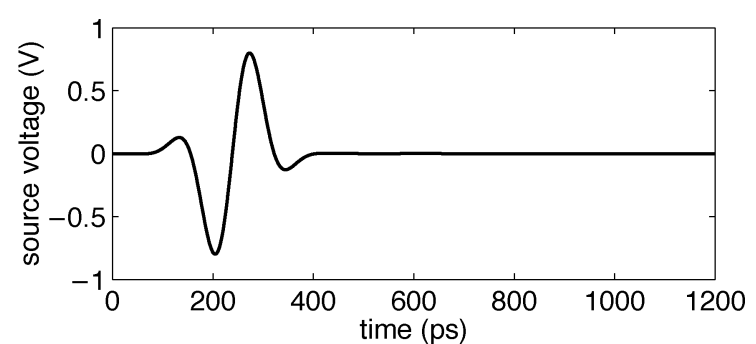

(a)

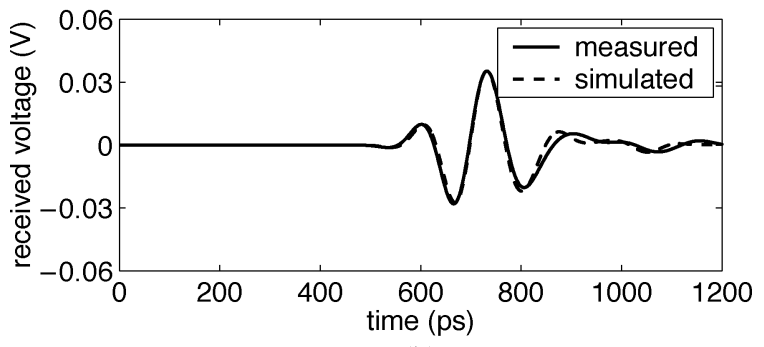

(b)

Fig. 5. (a) Source waveform applied to the input of the transmitting antenna (b) FDTD-computed and measured waveforms recorded at the receiving antenna located at a distance of $5 \mathrm{~cm}$ from the transmitter when both antennas are immersed in a medium matched to the normal breast tissue simulant.

fields radiated by the transmitting antenna at several observation points. Fig. 6(a) shows the radiated electric-field waveforms observed at a distance of $5 \mathrm{~cm}$ from the antenna over an angular span of $90^{\circ}$ on either side of boresight in the $E$-plane. Here, the $E$-plane intersects the launching plane and ridge and divides the antenna geometry into two symmetric halves. Fig. 6(b) shows the radiated field in the $H$-plane, which passes between the launching plane and ridge. The fidelity values calculated for the radiated waveforms within the antenna's main radiation lobe are greater than 0.8 .

\section{Signal Processing AND IMAge Formation}

After measuring backscatter signals $\left(S_{11}\right)$ from the multilayer breast phantoms, time-domain backscatter waveforms are synthesized. Next, dominant early-time artifacts are removed from the waveforms before 3-D MIST beamforming is employed to create an image of backscatter energy as a function of position.

The early-time artifacts in the received waveforms include antenna reverberation and reflections from the skin-breast interface. The data-adaptive algorithm reported in [22] is applied to remove these artifacts. In this algorithm, the artifact in the waveform received by a single antenna at one location is estimated as a filtered combination of the waveforms received at all other antenna locations and removed from the received waveform. The filter weights are chosen to minimize the residual signal meansquared error calculated over the artifact-dominated early-time response. We note that the artifact removal algorithm presented in [22] is not limited to a planar interface geometry. Surface curvature, variations in skin thickness, and heterogeneity of the underlying breast tissue are all accounted for by the data-adaptive procedure. The effectiveness of the algorithm was demonstrated with realistic 2-D numerical phantoms in [22].

Here, the efficacy of the artifact-removal algorithm is demonstrated using backscatter waveforms collected from
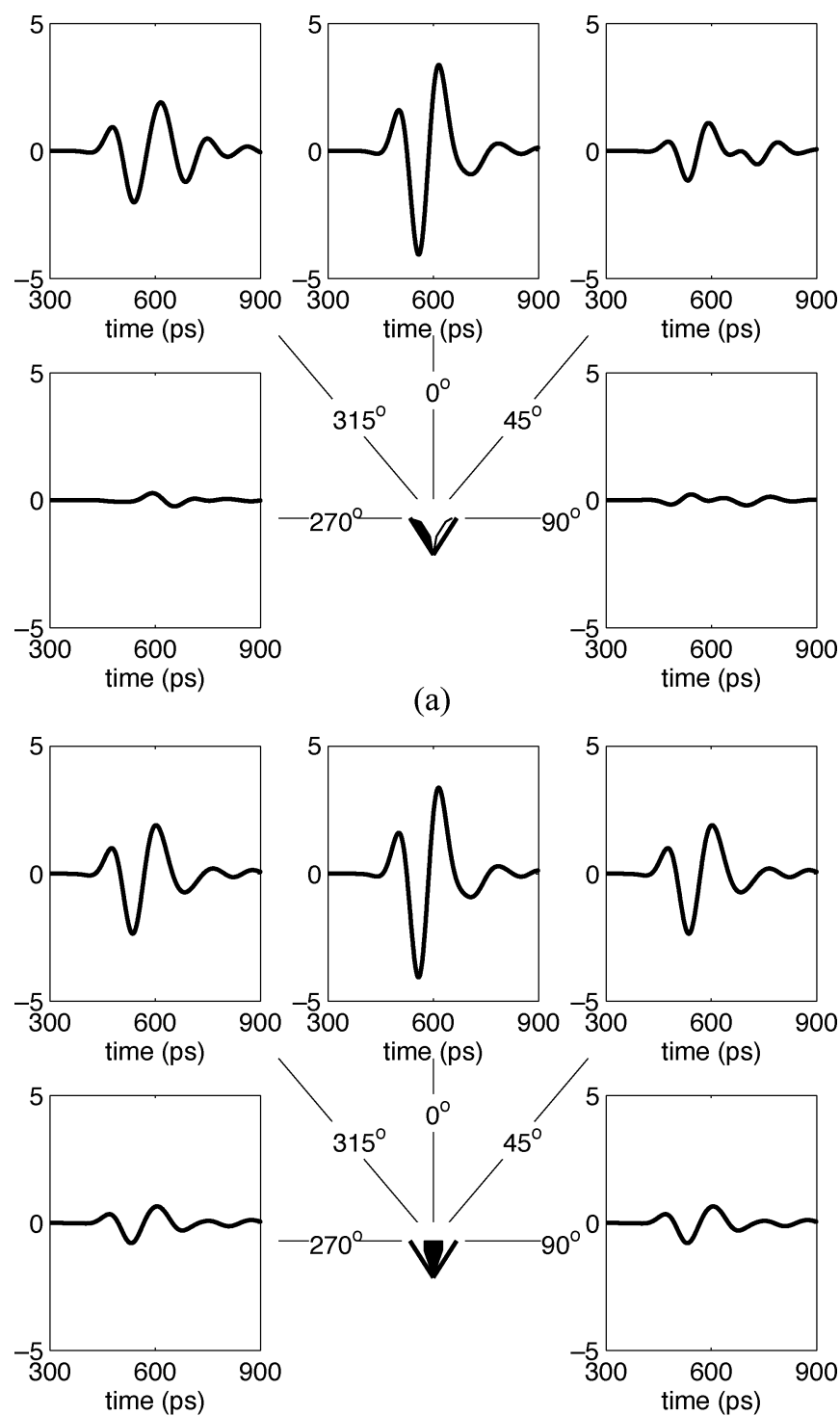

(b)

Fig. 6. Electric-field waveforms computed as a function of observation angle at a constant distance of $5 \mathrm{~cm}$ from the transmitting antenna when the antenna is immersed in soybean oil. (a) $E$-plane waveforms with the launching plane positioned on the right side. (b) $H$-plane waveforms.

the experimental breast phantom illustrated in Fig. 2. The 4-mm-diameter synthetic tumor made of simulant \#5 $\left(\varepsilon_{r, \text { malignant }}=5.2 \varepsilon_{r, \text { normal }}\right)$ is placed $2.0 \mathrm{~cm}$ below the skin surface under the central antenna location. In Fig. 7, the signals received at the central row in the antenna array are plotted before and after artifact removal. Prior to applying the artifact removal algorithm, the early-time response, shown by the dashed curves in the left-hand-side panel, is dominated by the antenna reverberation and skin-breast backscatter response. The late-time response, shown by the dashed curves in the right-hand-side panel using an enlarged vertical scale, contains the tumor response, which is completely masked by the slowly decaying artifact response. The solid curves represent the processed signals obtained by applying the artifact removal algorithm. The early-time artifact is almost completely eliminated as shown in the left-hand-side panel. The tumor response is now clearly evident in the late-time response depicted in the right-hand-side panel. The shaded areas highlight the time 


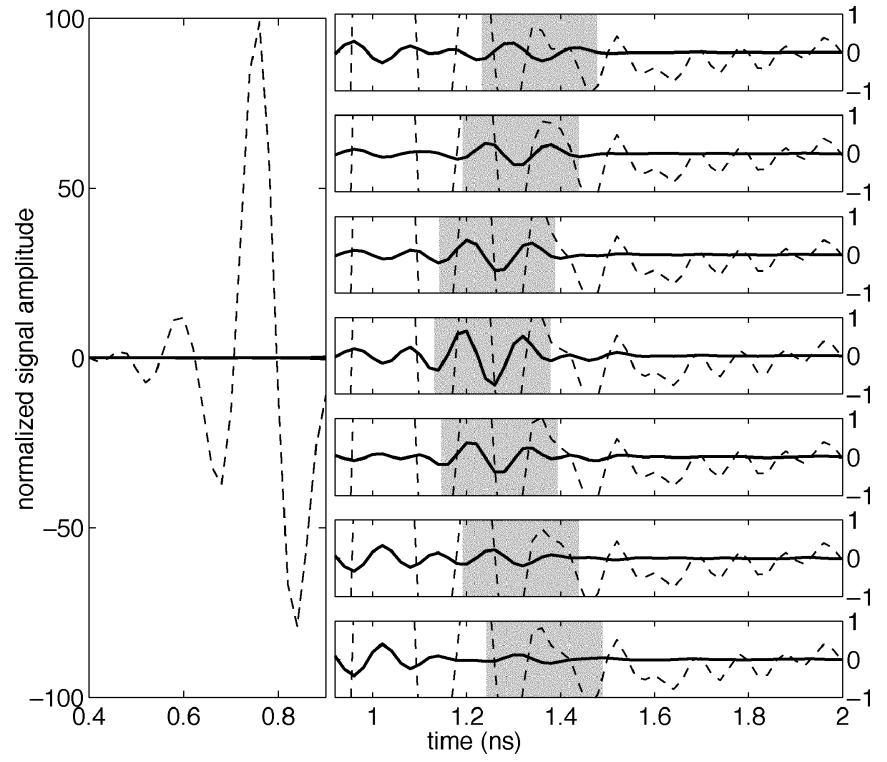

Fig. 7. Backscattered signals recorded for the experimental breast phantom with tumor simulant \#5. The waveforms received at the central row of the synthetic array are plotted before applying the artifact removal algorithm (dashed curves) and after (solid curves). The left-hand-side panel shows the early-time response, while the right-hand-side panel shows the late-time response. The shaded regions highlight the expected time window of the tumor response.

window in which the tumor response is expected based on the known material properties and location of the tumor.

After artifact removal, an image of backscatter energy is formed by applying space-time beamformers to the received backscatter, as proposed in [22] and [23]. For a candidate tumor location $\mathbf{r}_{0}$, beamforming serves to pass scattered signals that originate from $\mathbf{r}_{0}$ with approximate unit gain while attenuating other scattered signals and noise. This spatial discrimination is achieved by designing beamformer filters that solve a penalized least squares problem. In the beamformer design, backscatter from a tumor at $\mathbf{r}_{0}$ is modeled by an idealized point source in a homogeneous dielectric medium and propagation effects including path-length-dependent time delays, attenuation, and dispersion are equalized. By scanning the candidate tumor location over a 3-D reconstruction region and calculating the energy of the beamformer output at each scan location, we obtain an image of backscatter energy that we use to infer the presence and locations of strong scatterers. In a 2-D numerical study [22], we showed that this design is capable of detecting multiple small $(\leq 0.6 \mathrm{~cm})$ scatterers and is robust to imperfect knowledge of dielectric properties. In this paper, the beamformers are designed for our 3-D experimental setup using frequency-domain techniques [23]. The beamformer design assumes a $7 \times 7$ antenna array with $1-\mathrm{cm}$ lateral spacing centered above a $6 \mathrm{~cm} \times 6 \mathrm{~cm} \times 5 \mathrm{~cm}$ image reconstruction domain that is sampled with $1-\mathrm{mm}$ pixel resolution. The homogeneous dielectric medium assumed by the beamformers is matched to soybean oil.

Fig. 8 illustrates the ideal spatial discrimination capability of this 3-D beamformer. The beamformer gain, defined as the output power due to an idealized point scatterer in a homogeneous medium, is plotted on a decibel scale as a function of scat-

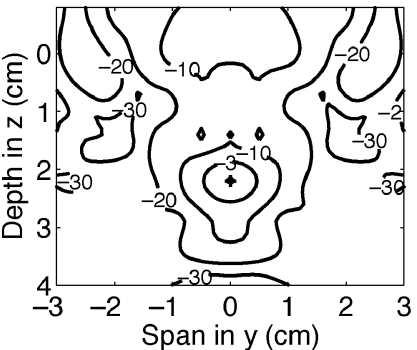

(a)

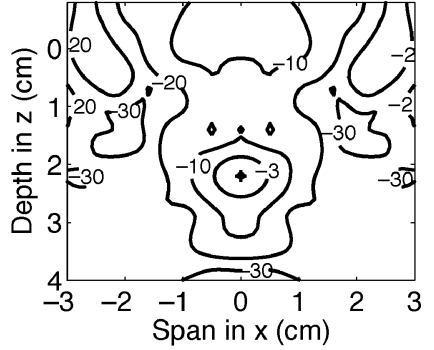

(b)

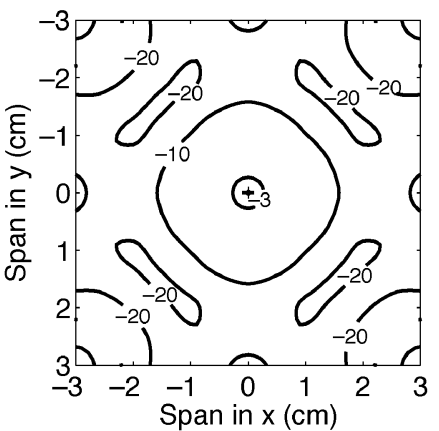

(c)

Fig. 8. Beamformer gain as a function of position when the design location is positioned $3.2 \mathrm{~cm}$ below the center of the antenna array. The coordinates are defined so that the array located at $z=-1 \mathrm{~cm}$. (a) $y z$-plane at $x=0.0 \mathrm{~cm}$. (b) $x z$-plane at $y=0.0 \mathrm{~cm}$. (c) $x y$-plane at $z=2.2 \mathrm{~cm}$. In each pattern, the location of the maximum is equal to the design location and is marked by a "+."

terer position in three orthogonal planes cutting through the design location (marked by "+"). These patterns indicate that the beamformer attenuates scattered signals originating from any location that is greater than $2 \mathrm{~cm}$ away from the design location by over $10 \mathrm{~dB}$.

Fig. 9 shows the MIST beamforming results for the experimental breast phantom consisting of 4-mm-diameter synthetic tumor placed $2 \mathrm{~cm}$ below the skin surface under the center of the array. Tumor simulant \#1 is used in this case to illustrate the results for the most challenging scenario of minimum malignant-to-normal tissue contrast $\left(\varepsilon_{r, \text { malignant }} / \varepsilon_{r, \text { normal }}=1.5\right)$. The 3-D image of backscatter energy is generated for a $6 \mathrm{~cm} \times$ $6 \mathrm{~cm} \times 5 \mathrm{~cm}$ domain with a 1-mm pixel resolution. Three orthogonal planes from the 3-D image are labeled using $x$ - and $y$-axes that correspond to the lateral dimensions of the imaging domain and a $z$-axis that corresponds to the depth dimension. The origin of the $z$-axis roughly corresponds to the location of the skin layer. The two energy peaks in the depth direction correspond to scattering from the top and bottom surfaces of the compact cylindrical tumor. The peak energy nearest the surface is located within $2 \mathrm{~mm}$ of the top edge of the actual tumor. For comparative purposes, the same beamforming process is also applied to the backscatter waveforms obtained from a tumor-free phantom. The signal-to-noise $(\mathrm{S} / \mathrm{N})$ ratio is defined as the ratio of the maximum tumor energy to the maximum noise energy in the tumor-free phantom. The tumor response in the received waveforms contributes to "signal," while "noise" is introduced by residual artifacts associated with antenna reverberation and reflections from the skin-breast interface, as well as conventional instrument noise. The $\mathrm{S} / \mathrm{N}$ is $4.9 \mathrm{~dB}$ for the reduced contrast scenario presented in Fig. 9. 


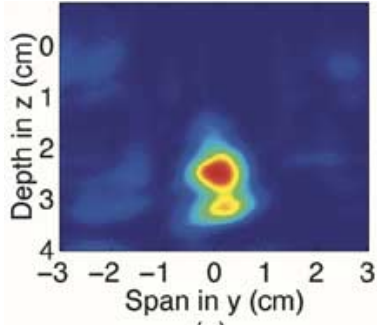

(a)

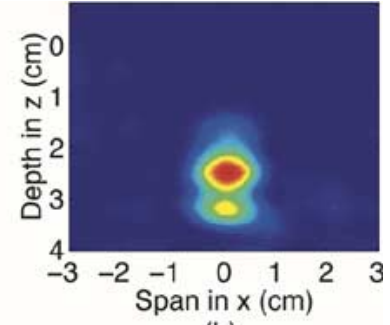

(b)

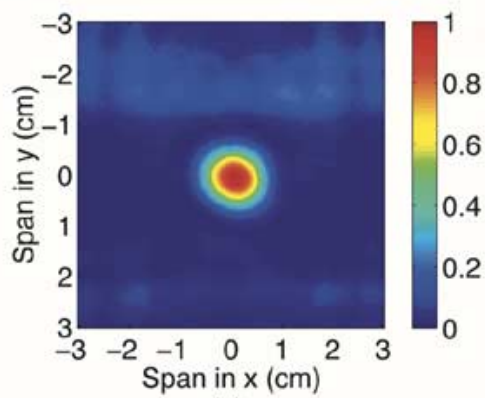

(c)

Fig. 9. Color image of backscattered energy for the experimental breast phantom of Fig. 2, which contains a 4-mm-diameter synthetic tumor located at a depth of $2 \mathrm{~cm}$ below the skin surface. The contrast in $\varepsilon_{r}$ between normal and malignant tissue simulants is only $1.5: 1$. The orthogonal planes intersect the shallower of the two energy peaks of the tumor response. (a) $y z$-plane at $x=0.1 \mathrm{~cm}$. (b) $x z$-plane at $y=0.1 \mathrm{~cm}$. (c) $x y$-plane at $z=2.3 \mathrm{~cm}$.

\section{INFLUENCE OF DieleCtric CONTRAST BETWEEN MALIGNANT AND NORMAL BREAST TISSUE}

This section presents a study of the effect of the dielectric contrast between malignant and normal breast tissue on the tumor backscatter response and the image S/N. As explained in Section III, the different dielectric contrasts are created using five tumor simulants with varying dielectric properties.

First, the effect of tissue contrast on the tumor response recorded by a single antenna element is examined. Five different dielectric properties for the tumor simulants are used in the experimental breast phantom. The time-domain waveform received at the antenna located at the center of the synthesized array is collected. The antenna reverberation and the reflection at the skin interface are removed by subtracting the antenna response obtained with a tumor-free phantom. Fig. 10(a) shows the measured tumor-response waveforms for five tumor simulants that yield contrasts ranging from 1.5:1 (tumor simulant \#1) to 5.2:1 (tumor simulant \#5). The tumor response, as expected, increases as the tissue contrast increases. In the case of the 5.2:1 dielectric contrast, the peak-to-peak tumor response is $1.3 \mathrm{mV}$ when the peak-to-peak voltage of the source pulse is $1.6 \mathrm{~V}$. Therefore, a minimum time-domain dynamic range of $62 \mathrm{~dB}$ is required to detect this tumor response. In the case of the minimum dielectric contrast $\left(\varepsilon_{r, \text { malignant }} / \varepsilon_{r, \text { normal }}=1.5\right)$, the peak-to-peak tumor response is $0.4 \mathrm{mV}$, which requires a dynamic range of $73 \mathrm{~dB}$ to be detected. This can be visualized in Fig. 10(b), where the dynamic-range requirements calculated from peak-to-peak tumor response values are plotted for all five cases as a function of contrast in dielectric constants between malignant and normal breast tissue simulants. Note that the dynamic range values quoted here are the minimum requirements for the corresponding contrast. Real decreases in

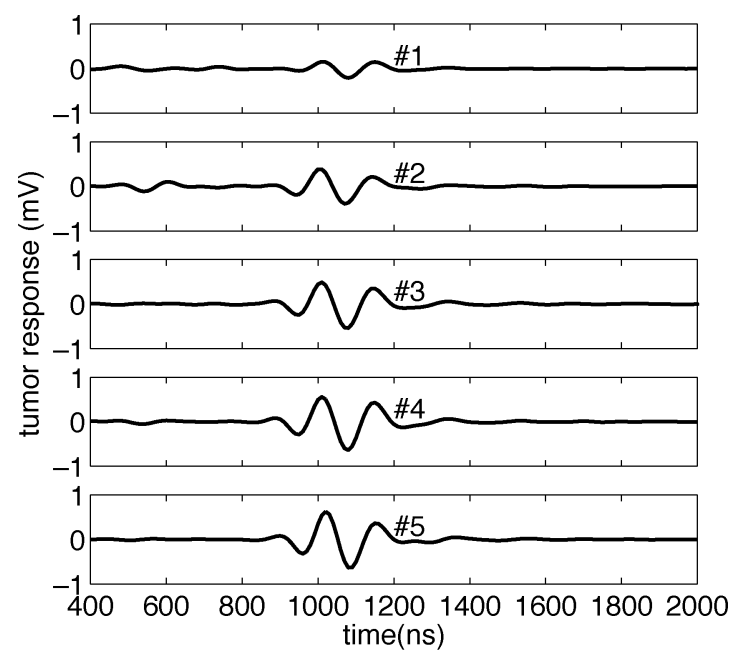

(a)

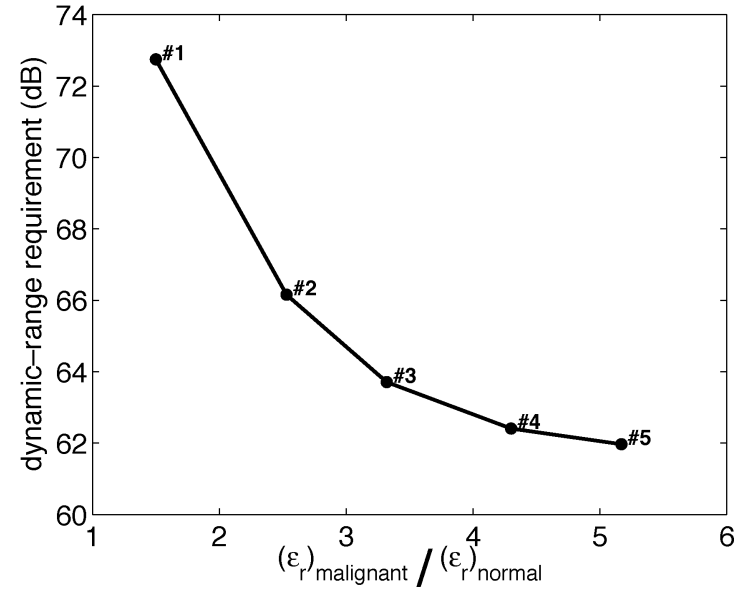

(b)

Fig. 10. (a) Measured backscatter waveforms from 4-mm synthetic tumors with malignant-to-normal tissue simulant contrasts ranging from 5.2:1 (tumor simulant \#5) to $1.5: 1$ (tumor simulant \#1). (b) Dynamic range requirements to capture the tumor responses as a function of the tissue contrasts.

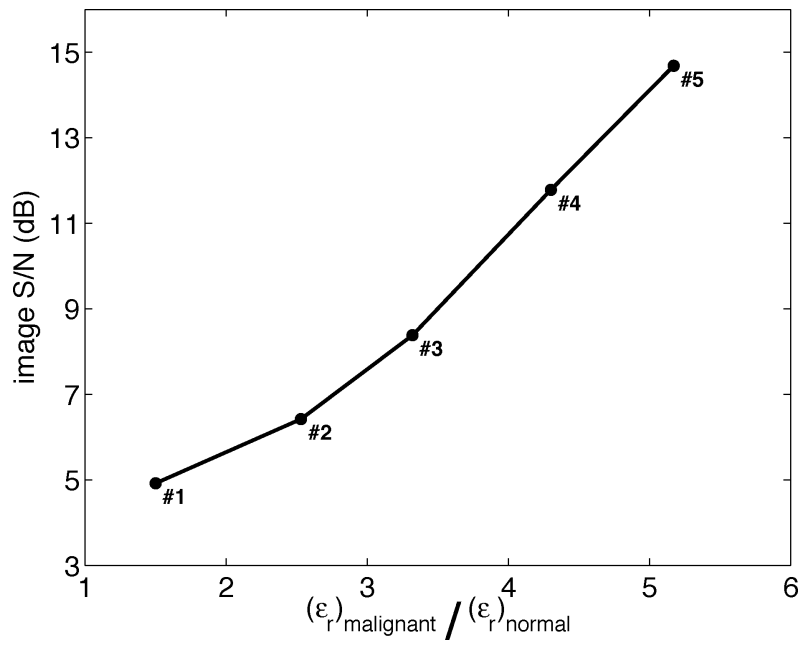

Fig. 11. Image $\mathrm{S} / \mathrm{N}$ as a function of the contrast in $\varepsilon_{r}$ between the malignant and normal breast tissue simulants.

malignant-to-normal breast tissue contrast are expected to arise from increases in normal breast tissue density, which also result in greater attenuation of both transmitted and backscattered microwave signals. 
The influence of malignant-to-normal dielectric contrast on image quality and tumor detectability is also studied. MIST beamforming results are obtained for the experimental breast phantom used in Section V, but with five different dielectric properties for the tumor simulants. Image $\mathrm{S} / \mathrm{N}$ is plotted in Fig. 11 as a function of contrast in $\varepsilon_{r}$ between normal and malignant tissue simulants. As the contrast increases from $1.5: 1$ to $5.2: 1$, the image $\mathrm{S} / \mathrm{N}$ improves from 4.9 to $14.5 \mathrm{~dB}$. Since the noise components are relatively constant given identical hardware and phantom geometry, the increase in tumor response translates directly to an improvement in image $\mathrm{S} / \mathrm{N}$.

\section{CONCLUSION}

We have presented the first experimental demonstration of 3-D MIST beamforming in multilayer breast phantoms with malignant-to-normal dielectric contrasts down to $1.5: 1$ for a 4-mm synthetic tumor. The enhanced focusing capabilities of MIST beamforming and the efficacy of a data-adaptive algorithm for removing antenna reverberation and reflections from the skin-breast interface have been fully demonstrated. Excellent agreement has been obtained between the experimental measurements and FDTD simulations of the UWB antenna used in the experiments. The influence of malignant-to-normal breast tissue dielectric contrast on the dynamic range requirements and tumor detectability has been summarized. Our experimental results suggest that MIST beamforming may offer the potential of detecting small breast tumors using state-of-the-art, but readily available hardware and robust signal-processing algorithms.

\section{ACKNOWLEDGMENT}

The authors would like to thank M. Choi, P. Gustafson, and L. Palmer, all with the Department of Electrical and Computer Engineering, University of Wisconsin-Madison, for their help with the experimental setup.

\section{REFERENCES}

[1] Mammography and Beyond: Developing Techniques for the Early Detection of Breast Cancer. Washington, DC: Inst. Med., Nat. Academy Press, 2000.

[2] P. T. Huynh, A. M. Jarolimek, and S. Daye, "The false-negative mammogram," Radiograph, vol. 18, no. 5, pp. 1137-1154, 1998.

[3] J. G. Elmore, M. B. Barton, V. M. Moceri, S. Polk, P. J. Arena, and S. W. Fletcher, "Ten-year risk of false positive screening mammograms and clinical breast examinations," New Eng. J. Med., vol. 338, no. 16, pp. 1089-1096, 1998.

[4] E. C. Fear, S. C. Hagness, P. M. Meaney, M. Okoniewski, and M. A. Stuchly, "Enhancing breast tumor detection with near field imaging," IEEE Microwave Mag., vol. 3, pp. 8-56, Mar 2002.

[5] K. L. Carr, "Microwave radiometry: Its importance to the detection of cancer," IEEE Trans. Microwave Theory Tech., vol. 37, pp. 1862-1869, Dec. 1989.

[6] B. Bocquet, J. C. van de Velde, A. Mamouni, Y. Leroy, G. Giaux, J. Delannoy, and D. Del Valee, "Microwave radiometric imaging at $3 \mathrm{GHz}$ for the exploration of breast tumors," IEEE Trans. Microwave Theory Tech., vol. 38, pp. 791-793, June 1990.

[7] S. S. Chaudhary, R. K. Mishra, A. Swarup, and J. M. Thomas, "Dielectric properties of normal and malignant human breast tissues at radiowave and microwave frequencies," Indian J. Biochem. Biophys., vol. 21, pp. 76-79, Feb. 1984

[8] A. J. Surowiec, S. S. Stuchly, J. R. Barr, and A. Swarup, "Dielectric properties of breast carcinoma and the surrounding tissues," IEEE Trans. Biomed. Eng., vol. 35, pp. 257-263, Apr. 1988.
[9] W. T. Joines, Y. Z. Dhenxing, and R. L. Jirtle, "The measured electrical properties of normal and malignant human tissues from 50 to $900 \mathrm{MHz}$," Med. Phys., vol. 21, pp. 547-550, Apr. 1994.

[10] R. A. Kruger, K. K. Kopecky, A. M. Aisen, D. R. Reinecke, G. A. Kruger, and W. L. Kiser, Jr., "Thermoacoustic CT with radio waves: A medical imaging paradigm," Radiology, vol. 211, pp. 275-278, 1999.

[11] L. V. Wang, X. Zho, H. Sun, and G. Ku, "Microwave-induced acoustic imaging of biological tissues," Rev. Sci. Instrum., vol. 70, pp. 3744-3748, 1999.

[12] P. M. Meaney and K. D. Paulsen, "Nonactive antenna compensation for fixed-array microwave imaging: Part II-Imaging results," IEEE Trans. Med. Imag., vol. 18, pp. 508-518, June 1999.

[13] P. M. Meaney, M. W. Fanning, D. Li, S. P. Poplack, and K. D. Paulsen, "A clinical prototype for active microwave imaging of the breast," IEEE Trans. Microwave Theory Tech., vol. 48, pp. 1841-1853, Nov. 2000.

[14] A. E. Souvorov, A. E. Bulyshev, S. Y. Semenov, R. H. Svenson, and G. P. Tatsis, "Two-dimensional computer analysis of a microwave flat antenna array for breast cancer tomography," IEEE Trans. Microwave Theory Tech., vol. 48, pp. 1413-1415, Aug. 2000.

[15] A. E. Bulyshev, S. Y. Semenov, A. E. Souvorov, R. H. Svenson, A. G. Nazarov, Y. E. Sizov, and G. P. Tatsis, "Computational modeling of three-dimensional microwave tomography of breast cancer," IEEE Trans. Biomed. Eng., vol. 48, pp. 1053-1056, Sept. 2001.

[16] Q. H. Liu, Z. Q. Zhang, T. T. Wang, J. A. Bryan, G. A. Ybarra, L. W. Nolte, and W. T. Joines, "Active microwave imaging I-2-D forward and inverse scattering methods," IEEE Trans. Microwave Theory Tech., vol. 50, pp. 123-133, Jan. 2002.

[17] S. C. Hagness, A. Taflove, and J. E. Bridges, "Two-dimensional FDTD analysis of a pulsed microwave confocal system for breast cancer detection: Fixed-focus and antenna-array sensors," IEEE Trans. Biomed. Eng., vol. 45, pp. 1470-1479, Dec. 1998.

[18] _ "Three-dimensional FDTD analysis of a pulsed microwave confocal system for breast cancer detection: Design of an antenna-array element," IEEE Trans. Antennas Propagat., vol. 47, pp. 783-791, May 1999.

[19] X. Li and S. C. Hagness, "A confocal microwave imaging algorithm for breast cancer detection," IEEE Microwave Wireless Comp. Lett., vol. 11, pp. 130-132, Mar. 2001.

[20] E. C. Fear and M. A. Stuchly, "Microwave detection of breast cancer," IEEE Trans. Microwave Theory Tech., vol. 48, pp. 1854-1863, Nov. 2000

[21] E. C. Fear, X. Li, S. C. Hagness, and M. Stuchly, "Confocal microwave imaging for breast cancer detection: Localization of tumors in three dimensions," IEEE Trans. Biomed. Eng., vol. 49, pp. 812-822, Aug. 2002.

[22] E. J. Bond, X. Li, S. C. Hagness, and B. D. Van Veen, "Microwave imaging via space-time beamforming for early detection of breast cancer," IEEE Trans. Antennas Propagat., vol. 51, pp. 1690-1705, Aug. 2003.

[23] S. K. Davis, E. J. Bond, X. Li, S. C. Hagness, and B. D. Van Veen, "Microwave imaging via space-time beamforming for early-stage breast cancer detection: Beamformer design in the frequency domain," J. Electromagn. Waves Applicat., vol. 17, no. 2, pp. 357-381, 2003.

[24] E. C. Fear, J. Sill, and M. A. Stuchly, "Experimental feasibility study of confocal microwave imaging for breast tumor detection," IEEE Trans. Microwave Theory Tech., vol. 51, pp. 887-892, Mar. 2003.

[25] X. Li, E. J. Bond, S. C. Hagness, B. D. Van Veen, and D. van der Weide, "Three-dimensional microwave imaging via space-time beamforming for breast cancer detection," presented at the IEEE AP-S Int. Symp. and USNC/URSI Radio Science Meeting, San Antonio, TX, June 2002.

[26] A. Taflove and S. Hagness, Computational Electrodynamics: The FiniteDifference Time-Domain Method, 2nd ed. Boston, MA: Artech House, 2000.

[27] K. R. Foster and H. P. Schwan, "Dielectric properties of tissues and biological materials: A critical review," Crit. Rev. Biomed. Eng., vol. 17, pp. 25-104, 1989.

[28] S. Gabriel, R. W. Lau, and C. Gabriel, "The dielectric properties of biological tissues: III. Parametric models for the dielectric spectrum of tissues," Phys. Med. Biol., vol. 41, pp. 2271-2293, 1996.

[29] S. S. Stuchly, C. L. Sibbald, and J. M. Anderson, "A new aperture admittance model for open-ended waveguides," IEEE Trans. Microwave Theory Tech., vol. 42, pp. 192-198, Feb. 1994.

[30] K. L. Walton and V. C. Sundberg, "Broadband ridged horn design," Microwave J., vol. 4, no. 2, pp. 96-101, Apr. 1964.

[31] B. M. Notaros, C. D. McCarrick, and D. P. Kasilingam, "Two numerical techniques for analysis of pyramidal horn antennas with continuous metallic ridges," in IEEE AP-S Int. Symp., vol. 2, 2001, pp. 560-563.

[32] E. T. Rosenbury, G. K. Burke, S. D. Nelson, R. D. Stever, G. K. Gorverno, and D. J. Mullenhoff, "Low cost impulse compatible wideband antenna," U.S. Patent 6348898 
[33] X. Li, S. C. Hagness, M. K. Choi, and D. W. van der Weide, "Numerical and experimental investigation of an ultrawide-band ridged pyramidal horn antenna with curved launching plane for pulse radiation," IEEE Antennas Wireless Propagat. Lett., vol. 2, no. 18, pp. 259-262, 2003.

[34] M. Piket-May, A. Taflove, and J. Baron, "FD-TD modeling of digital signal propagation in 3-D circuits with passive and active loads," IEEE Trans. Microwave Theory Tech., vol. 42, pp. 1514-1523, Aug. 1994.

[35] J. P. Berenger, "A perfectly matched layer for the absorption of electromagnetic waves," J. Comput. Phys., vol. 114, pp. 185-200, 1994.

[36] O. E. Allen, D. A. Hill, and A. R. Ondrejka, "Time-domain antenna characterizations," IEEE Trans. Electromagn. Compat., vol. 35, pp. 339-345, Aug. 1993.

[37] B. Van Veen, "Minimum variance beamforming," in Adaptive Radar De tection and Estimation, S. Haykin and A. Steinhardt, Eds. New York: Wiley, 1992, ch. 4, pp. 161-236.

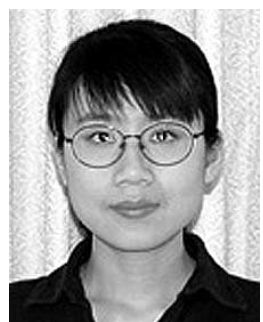

Xu Li (S'99-M'03) received the B.S. degree in biomedical engineering from Tsinghua University, Beijing, China, in 1998, and the M.S. degree in biomedical engineering and $\mathrm{Ph} . \mathrm{D}$. degree in electrical and computer engineering from the University of Wisconsin-Madison, in 2000 and 2003, respectively.

Since October of 2003, she has been a Post-Doctoral Fellow with the Department of Biomedical Engineering, Northwestern University, Evanston, IL. Her current research interests include applied and computational electromagnetics, bioelectromagnetics, and biophotonics.

$\mathrm{Dr}$. Li is a member of the Bioelectromagnetics Society and the Optical Society of America. She was the recipient of a Wisconsin Alumni Research Foundation Graduate Fellowship presented by the University of Wisconsin-Madison in 1998. She was also the recipient of the Student Paper Competition Second Prize Award presented at the 2003 IEEE Microwave Theory and Techniques Society (IEEE MTT-S) International Microwave Symposium (IMS).

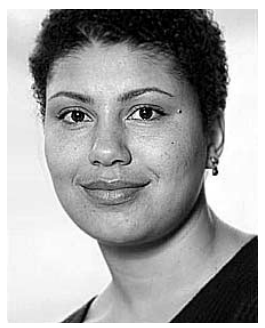

Shakti K. Davis (S'99) received the B.S. degree from New Mexico State University, Las Cruces, in 1999, the M.S. degree from the University of Wisconsin-Madison, in 2001, both in electrical engineering, and is currently working toward the Ph.D degree in electrical engineering at the University of Wisconsin-Madison

Her research interests include biomedical applications of signal processing.

Ms. Davis was the recipient of a University of Wisconsin Graduate Engineering Research Scholars Fellowship and is currently supported by a National Institutes of Health (NIH) Minority Pre-Doctoral Fellowship.

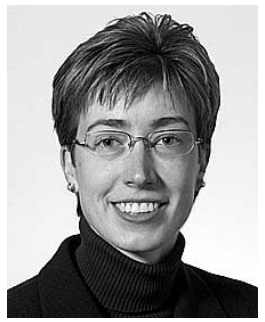

Susan C. Hagness (S'91-M'98) received the B.S. (with highest honors) and Ph.D. degree in electrical engineering from Northwestern University, Evanston, IL, in 1993 and 1998, respectively. While working toward the Ph.D. degree, she was a Nationa Science Foundation (NSF) Graduate Fellow and a Tau Beta Pi Spencer Fellow.

Since August 1998, she has been with the University of Wisconsin-Madison, where she is currently an Associate Professor with the Department of Electrical and Computer Engineering and a faculty affiliate with the Department of Biomedical Engineering. Her research interests include FDTD theory and applications, microwave imaging and sensing techniques for biological and medical applications, and full-wave analysis and design of photonic microstructures. She coauthored Computational Electrodynamics: The Finite-Difference Time-Domain Method. (Boston, MA: Artech House, 2000, 2nd ed.).

Dr. Hagness is a member of the IEEE Antennas and Propagation Society (IEEE AP-S) Administrative Committee (AdCom). She is currently an associate editor for the IEEE ANTENNAS AND WIRELESS PROPAGATION LETTERS She was an invited participant in the National Academy of Engineering's Frontiers of Engineering Symposium in 2002. She was the recipient of the Presidential Early Career Award for Scientists and Engineers presented by the White House in 2000. She was the recipient of the Booker Fellowship Award presented by the United States National Committee of the International Union of Radio Science in 2002. She was also named one of the 100 top young innovators in science and engineering in the world by the Massachusetts Institute of Technology (MIT) Technology Review magazine. In 2003, she was the recipient of the University of Wisconsin Emil Steiger Distinguished Teaching Award.

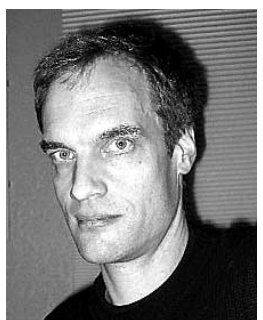

Daniel W. van der Weide (S' $86-\mathrm{M}^{\prime} 86$ ) received the B.S.E.E. degree from the University of Iowa, Iowa City, in 1987, and the Master's and Ph.D. degrees in electrical engineering from Stanford University, Stanford, CA, in 1989 and 1993, respectively.

He held summer positions with the Lawrence-Livermore National Laboratory and Hewlett-Packard, and full-time positions with Motorola as an Engineer and the Watkins-Johnson Company as a Member of the Technical Staff. From 1993 to 1995, he was a Post-Doctoral Researcher with the Max-Planck-Institut für Festkörperforschung (Solid State Research), Stuttgart, Germany, after which he joined the Department of Electrical and Computer Engineering, University of Delaware, as an Assistant and Associate Professor and Director of the Center for Nanomachined Surfaces. In 1999, he joined the Department of Electrical and Computer Engineering, University of Wisconsin-Madison, as an Associate Professor. He is a University of Wisconsin Vilas Associate for 2002-2004. He is the Principal Investigator on a 2003 Air Force Office of Scientific Research (AFOSR) Multiuniversity Research Initiative (MURI) overseen by Lt. Col. G. Pomrenke entitled, "Nanoprobe Tools for Molecular Spectroscopy and Control." His current research involves ultrafast electronics, low-dimensional electron systems, and the application of high-frequency techniques in biotechnology.

Dr. van der Weide was the recipient of the National Science Foundation (NSF) CAREER and PECASE Awards in 1997 and the Office of Naval Research (ONR) Young Investigator Program Award in 1998.

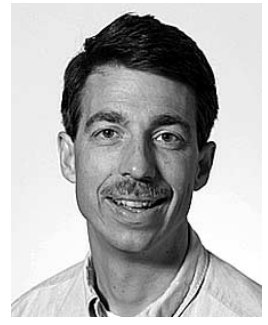

Barry D. Van Veen (S'81-M'86-SM'97-F'02) was born in Green Bay, WI. He received the B.S. degree from the Michigan Technological University, Houghton, in 1983, and the Ph.D. degree from the University of Colorado at Boulder, in 1986, both in electrical engineering. He was an Office of Naval Research (ONR) Fellow while working toward the Ph.D. degree.

In Spring 1987, he was with the Department of Electrical and Computer Engineering, University of Colorado at Boulder. Since August 1987, he has been with the Department of Electrical and Computer Engineering, University of Wisconsin-Madison, where he is currently a Professor. He coauthored Signals and Systems (New York: Wiley, 1999, 1st ed.; 2003, 2nd ed.). His research interests include signal processing for sensor arrays, wireless communications, and biomedical applications of signal processing.

Dr. Van Veen was an associate editor for the IEEE TRANSACTIONS ON Signal Processing and the IEEE Signal Processing Society's Statistical Signal and Array Processing Technical Committee and the Sensor Array and Multichannel Technical Committee. He was a recipient of a 1989 Presidential Young Investigator Award presented by the National Science Foundation and a 1990 IEEE Signal Processing Society Paper Award. He was also the recipient of the Holdridge Teaching Excellence Award presented by the Department of Electrical and Computer Engineering, University of Wisconsin-Madison, in 1997. 